\title{
A novel concept for the manufacture of individual sapphire-metallic hip joint endoprostheses
}

\author{
A.G. Mamalis, ${ }^{1, *}$ J.J. Ramsden, ${ }^{2}$ A.I. Grabchenko, ${ }^{3}$ L.A. Lytvynov, ${ }^{4}$ V.A. Filipenko ${ }^{5}$ and S.N. Lavrynenko ${ }^{3}$ \\ ${ }^{1}$ Laboratory of Manufacturing Technology, National Technical University of Athens, Greece \\ ${ }^{2}$ Advanced Materials Department, Cranfield University, UK \\ ${ }^{3}$ Department of Integrated Technologies in Mechanical Engineering, National Technical University Kh. P. I., Kharkov, Ukraine \\ ${ }^{4}$ Institute for Single Crystals, National Academy of Science of Ukraine, Kharkov, Ukraine \\ ${ }^{5}$ Sytenko Institute of Spine and Joint Pathology, Ukrainian Academy of Medical Sciences, Kharkov, Ukraine
}

\begin{abstract}
At the present time, artificial joints made with metallic, ceramic, metal-polymeric or ceramicpolymeric friction pairs substituting for the natural biomechanic articulations "head of the hip joint-acetabulum" are widely used for endoprosthetic operations on hip joints. Experience gained in the course of more than 2000 operations has shown that along with the advantageous properties of modern endoprosthetic constructions made of metal, ceramics and polymers, they have certain drawbacks. Among them are insufficient biological inertness and susceptibility to excessive wear of the friction pair components. In addition, as a result of wear of the hinge friction pair, toxic and oncologically dangerous products of degradation accumulate in the different organs and tissues. This in turn results in severe complications and demands correspondingly complicated corrective intervention, often leading to worse disability than that which the original operation was designed to cure. The aim of the study reported here was the development and clinical validation of a highly effective and long-lived hip joint endoprosthesis with a sapphire head whose wear capacity is superior to all others. The endoprosthesis consists of a metallic pedicle, a dismountable articulation (metallic necklayer of supramolecular polyethylene-sapphire head) and an acetabular cup. The endoprostheses with the sapphire head proved themselves positively in clinical trials and are considered to be highly promising for future applications.
\end{abstract}

Keywords: endoprostheses, sapphire, rapid prototyping

\section{INTRODUCTION}

During the last decade, hip joint endoprostheses have been ever more widely carried out in orthopaedic practice in the world; more than 400000 operations are performed every year and more than 100 types of endoprosthetic construction have been designed $[1,2]$. The best known are those of Biomet, Zimmer (USA), and Aesculap (Germany), in which metallic and polymer or ceramic and polymer joints are used.

Analysis of the results of endoprosthetic operations on joints show that in $7-12 \%$ of cases $2-3$ years later, and in $25-30 \%$ of cases $5-7$ years later, complications arise that are generally deleterious to the health of the recipient [3]. The most frequent and dangerous complication is an aseptic shattered condition of the endoprosthesis in the bone tissue, which leads to the necessity of recurrent operations.

One of the negative factors leading to this condition is friction in the hinge of the artificial joint, releasing nanoparticles produced from the endoprosthetic material. Furthermore, different loads in the hinge of the endoprosthesis during walking cause friction in the components of the joint and it wears out (see Fig. 1).

"Corresponding author. E-mail: mamalis@central.ntua.gr
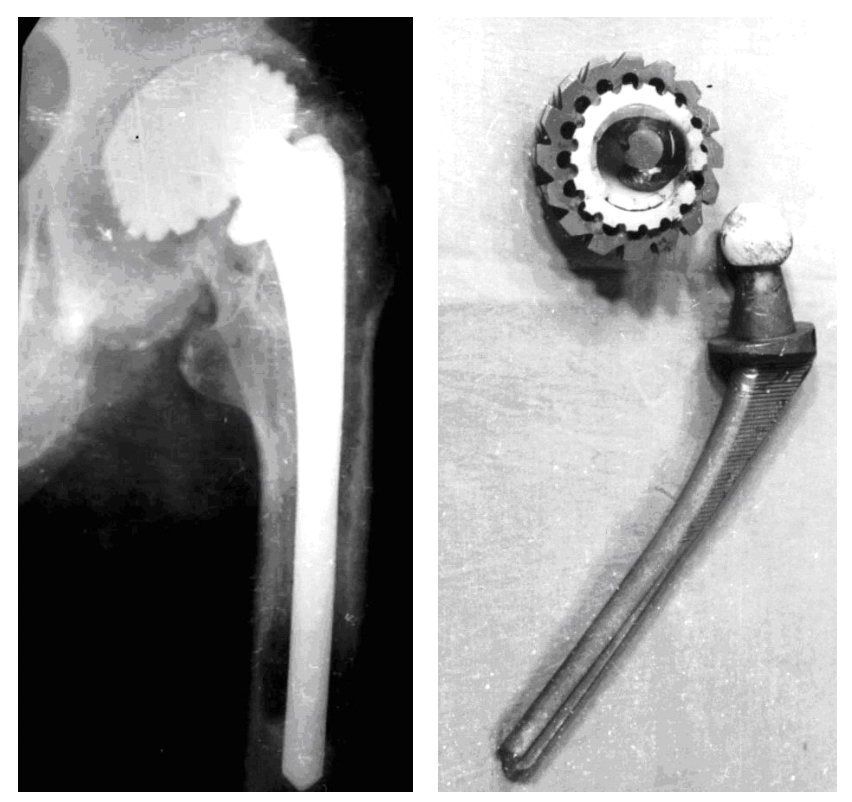

Figure 1. Wear of the metallic head and ferulene bush of a normal endoprosthesis after 10 years of operation.

The products of the action of the friction are toxic, and are accumulated in various important body organs (liver, heart, bone marrow etc.). This accumulation can provoke serious oncological diseases. 
Hence, there is a general search for new tribologically and biologically inactive materials for the creation of better artificial joints [4-6]. At the Kharkov Scientific Research Institute of Spine and Joint Pathology (Sytenko Institute), endoprosthetic operations on the hip joint have been performed during the past 25 years and more than 1000 patients have been operated on, while research work on improving endoprosthetic constructions and operative methods is being continuously undertaken.

In particular, the Sytenko Institute has pioneered the creation of a new endoprosthesis using the sapphiresapphire pair for the hinge. Such a pair is characterized by a practically zero coefficient of friction and the absence of any gradual weakening of the material in its implantation environment. This makes it possible to achieve stable fixation of the implant in the human bone marrow over a long interval.

Experience over the last 10 years acquired by working with many other types of sapphire implants has demonstrated their unparalleled inertness and biocompatibility. It is this experience that enables us now to move to hip joint endoprostheses with sapphire pairs, which is one of the most complicated implants.

2. SOLUTIONS TO THE MEDICAL-TECHNOLOGICAL PROBLEMS ARISING IN THE MANUFACTURING PROCESS OF INDIVIDUAL STEMS FOR SAPPHIRE-METALLIC HIP JOINT ENDOPROSTHESES AND SPECIAL RASPS FOR THIGH BONE CHANNEL FITTING AFTER COMPUTER TOMOGRAPHY, USING RAPID PROTOTYPINGTECHNOLOGY

The hip joint endoprosthesis is now a routine operation, which is executed by practically any orthopaedic hospital worldwide. However, the aseptic instability of the components of the prosthetic device, especially the stem, is an unsolved problem [8].

The development of early instability is connected with the replacement of a proximal part of the thigh bone and its substitution by an artificial implant. The residual part of the bone may then change its characteristics under excessive loads. This can result in destruction of the bone tissue faster than bone formation, which is one of the main reasons for the emergence of early instability in the implant [9].

Stable primary fixation of the prosthesis stem without cement is necessary for prophylaxis of this complication. To this end, there are certain methods for ensuring a correct disposition of the implant in the bone channel; and selection of the form and size of the prosthesis stem so as to be in correspondence with the form and size of the channel in the thigh bone is necessary, thus ensuring the greatest possible contact of the implant surface with the bone tissue. According to our data, this should be not less than $60 \%$ of the total implant surface. This is achieved by processing the thigh bone channel with a special rasp, whose dimensions should correspond to standard forms and sizes of the implant stem $[9,10]$.

Another problem arises because each person has individual features, including the structure of the thigh bone channel. Usually a standard rasp is used to fit the individual channel to the standard endoprosthesis stem. Commonly this procedure is excessively traumatic and results in unnecessary ablation of healthy bone tissues. Moreover, the violent modification of the form of the thigh bone channel also results in unpredictable modifications to the load-bearing surface, and the patient requires a long rehabilitation period for adaptation to the new biomechanical situation [10].

The optimum solution to this problem is the use of an individual endoprostheses with individual stems. Sometimes individual endoprostheses, such as artificial limbs, may be produced based on X-ray images of the hip joint in two projections, or from computer tomography; the latter delivers more points and hence allows more precise and accurate manufacturing [11]. Today, a few companies are indeed manufacturing individual endoprostheses using three-dimensional mathematical models together with specialized software. After the computer tomography, a virtual model of the hip joint with the individual stem is created. The model is then transferred to a robot, which produces the endoprosthesis by cutting from the selected material. However, the transition from the virtual model to a finished product is an extremely laborious and expensive process requiring much time and material $[12,13]$. At the same time, a patient must await the completion of the lengthy procedure for manufacturing such an implant. Hence, we have developed methods for the rapid preparation of all the necessary software and operating programs for manufacturing of both the endoprosthesises with individual stems, and the special individual rasps for fitting the thigh bone channel after computer tomography and creation of a 3D model. The method is based on rapid prototyping technology. We have obtained good results in implanting metallic-sapphire endoprostheses with Ukrainian patients.

\section{GROWING SPECIAL ORIENTED SAPPHIRE MONOCRYS- TALS, MANUFACTURING THE HEADS OF THE SAPPHIRE- METALLIC HIP JOINT ENDOPROSTHESES, AND THE COMPARISON OF THEIR TRIBOLOGICAL CHARACTERIS- TICS IN COMPARISON WITH CO-MO-CR AND $\alpha-\mathrm{AL}_{2} \mathrm{O}_{3}$ - BASED CERAMICS}

The sapphire friction pairs were made from a supply of highly purified initial materials, and the crystals grown at $2100^{\circ} \mathrm{C}$ in vacumo. Under such conditions additional purification of the material takes place and the content of the main substance (aluminum oxide) achieved is $99.99 \%$. 
The coefficient of friction was controlled by varying the concentration of cation and anion vacancies, by varying the redox potential within the sapphire baking zone.

The nature of the materials selected for the movable articulations of the artificial joints considerably influence their working capacity and their life. The materials for endoprostheses must be chemically inert, non-toxic and biocompatible with the human organs with which they come into contact. In addition they must possess an optimal complex of physico-mechanical properties including high strength and mechanical resistance as well as a low friction coefficient. In modern joint endoprosthetics, Co-based alloys and $\alpha-\mathrm{Al}_{2} \mathrm{O}_{3}$-based ceramics applied in combination with high molecular weight polyethylene are considered to be the standard materials. The wear resistance of these materials has been insufficiently studied; however there are indications in the literature that the wear is 100 to $200 \mu \mathrm{m} /$ year for the ceramic $\mathrm{Al}_{2} \mathrm{O}_{3}$ /polyethylene and metal/polyethylene pairs. However, data for the friction coefficients are almost wholly absent.

We simulated the slipping friction to which the actual prosthesis would be expected to be subject in the UTI tribometer developed by SCTB FTINT, National Academy of Sciences of Ukraine, according to the "disk/upon disk" scheme. The magnitude of the friction interaction was selected in accord with load velocity parameter modelling of the friction elements of real joints. A loading from 200 to $1000 \mathrm{~N}$ was applied perpendicular to the surface face of the disks, the slipping velocity being $0.5 \mathrm{~m} / \mathrm{s}$.

One of the elements of the friction pair was made from an Co-Mo-Cr alloy, an $\alpha-\mathrm{Al}_{2} \mathrm{O}_{3}$-based ceramic or a sapphire monocrystal, and the other element from supramolecular polyethylene already permitted to be used in medicine.

Fig. 2 shows that the friction behaviours of the CoMo-Cr/polyethylene and ceramic/polyethylene friction pairs have some distinguishing features. In the initial stage (period of adjustment), the friction pair incorporating CoMo-Cr show a gradual increase of the friction coefficient up to $f=0.038$, whereas the ceramic/polyethylene friction pair shows a high value of the friction coefficient at the moment of starting adjustment $(f=0.14)$ with a further gradual decrease up to $f=0.1$. Further stabilization of the friction coefficient at the level of the values that are usually observed for antifriction materials $(f=0.06$ to $0.08)$ then occurs. The dynamics of the friction coefficient when using the sapphire monocrystal as one of the elements is similar to that observed for the Co-Mo-Cr/ polyethylene friction pair.

At given values of the load-velocity parameters, all the pairs of materials studied have low values of the friction coefficient and are characterized by a certain stability of the friction behaviour (Figs 3-5).

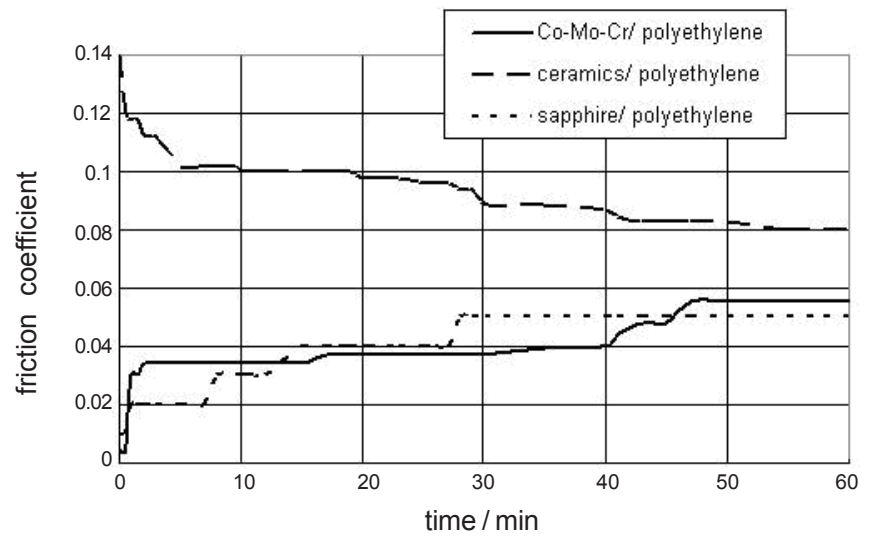

Figure 2. Change of friction coefficient over time for different combinations of materials (see text for details).

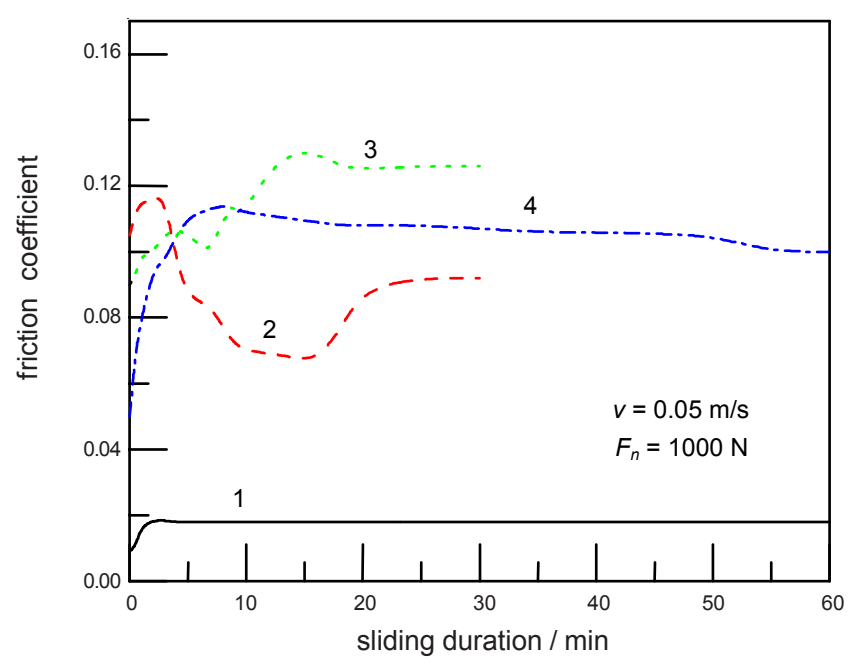

Figure 3. Dynamics of changes of friction factor during sliding for various pairs with a high normal load $\left(v=0.05 \mathrm{~m} / \mathrm{s}, F_{n}=1000 \mathrm{~N}\right)$. 1, Co-Cr-Mo/polyethylene; 2 , Ti alloy/Ti alloy; 3 , sapphire/ ruby; 4 , sapphire/sapphire.

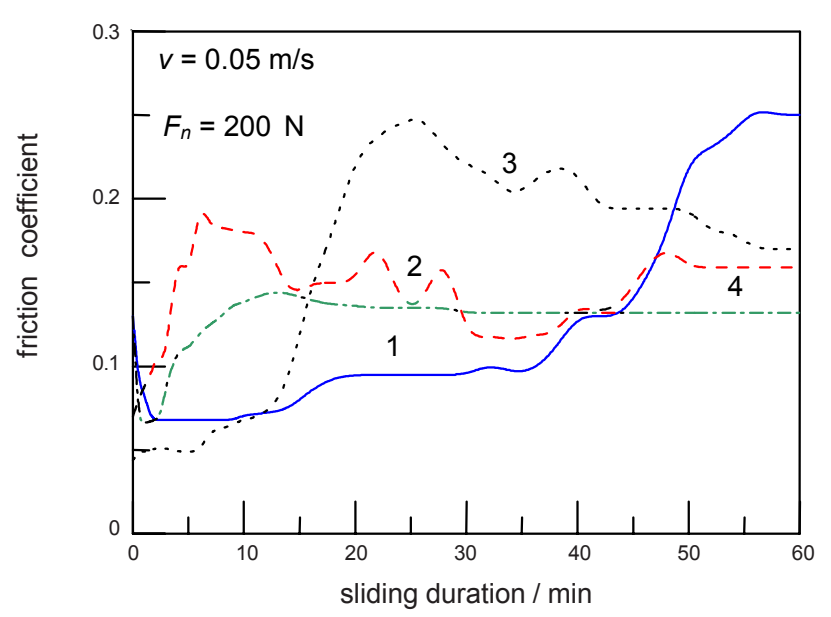

Figure 4. Influence of crystallographic orientation of contact surface on friction coefficient for sapphire/sapphire pairs $\left(v=0.05 \mathrm{~m} / \mathrm{s}, F_{n}=200 \mathrm{~N}\right) .1,(0001) ; 2,\{10 \overline{1} 0\} ; 3,\{11 \overline{2} 0\} ;$ $4,\{10 \overline{1} 2\}$. Contact surface of upper specimen: basic planar surface (0001). 


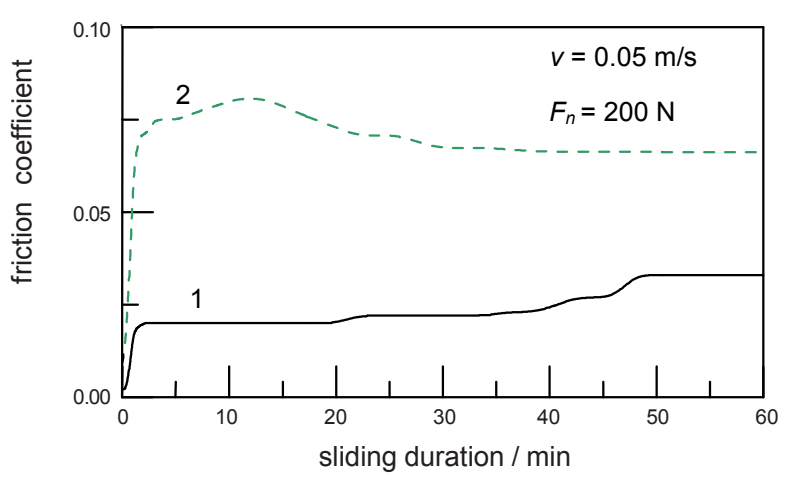

Figure 5. Comparison of frictional behaviour of "standard"and monocrystalline friction pairs in imitation of the influence of synovial fluid $\left(v=0.05 \mathrm{~m} / \mathrm{s}, F_{n}=200 \mathrm{~N}\right) .1$, Co-Cr-Mo alloy/ polyethylene; 2 , sapphire (0001)/sapphire $\{10 \overline{1} 2\}$.

Under conditions of certain crystallographic orientations, the contacting surfaces of the sapphire friction pairs have a rather low and stable friction coefficient $(0.05-0.10)$ as well as an extraordinarily high wear capacity. The decreased friction and wear in combination with high biochemical inertness and biological compatibility make the sapphire friction pair highly attractive for endoprosthetics.

\section{SAPPHIRE ENDOPROSTHESES IN CLINICAL PRACTICE}

Ultraprecision machining allows spheres to be manufactured with a sphericity error below $2 \mu \mathrm{m}$. Physicomechanical tests of the sapphire head and dismountable articulation (metallic neck-supramolecular polyethylene layer-sapphire head) showed a sufficient margin of strength (more than $50 \mathrm{kN}$ ).

Physicomechanical-medicobiological investigations of the sapphire showed that this material has a high grade of biocompatibility with human organisms.

The construction of endoprostheses with sapphire heads (see Figs 6-8) was used in the clinic of the Sytenko Institute of Spine and Joint Pathology, for 5 patients with grade III coxarthrosis. The method of the operation was developed in the clinic. Remote postoperative results were assessed after 5 years. In all the cases positive results were obtained, i.e. the locomotorium function of the joints was restored in the patients operated upon.

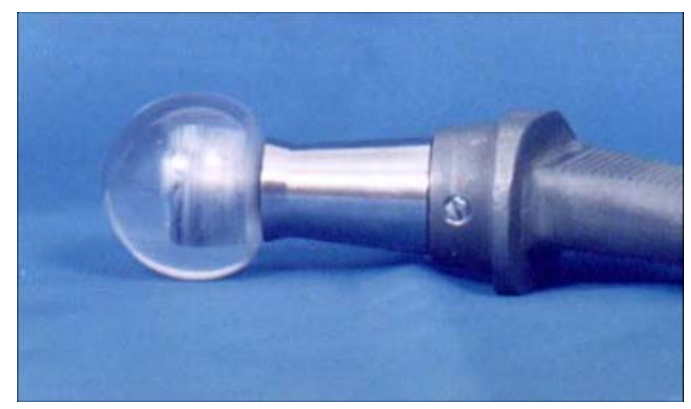

Figure 6. Hip joint endoprosthesis with a sapphire head.

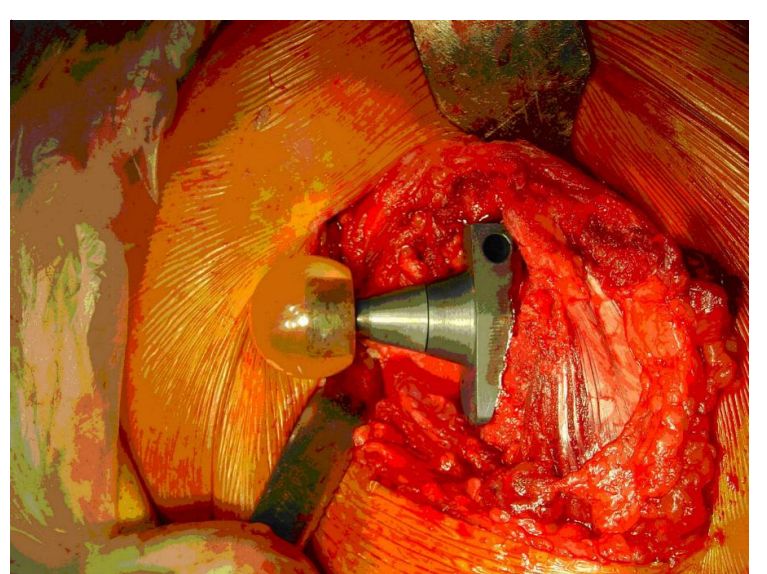

Figure 7. An intermediate stage of an operation on a hip joint using an endoprosthesis with a sapphire head.
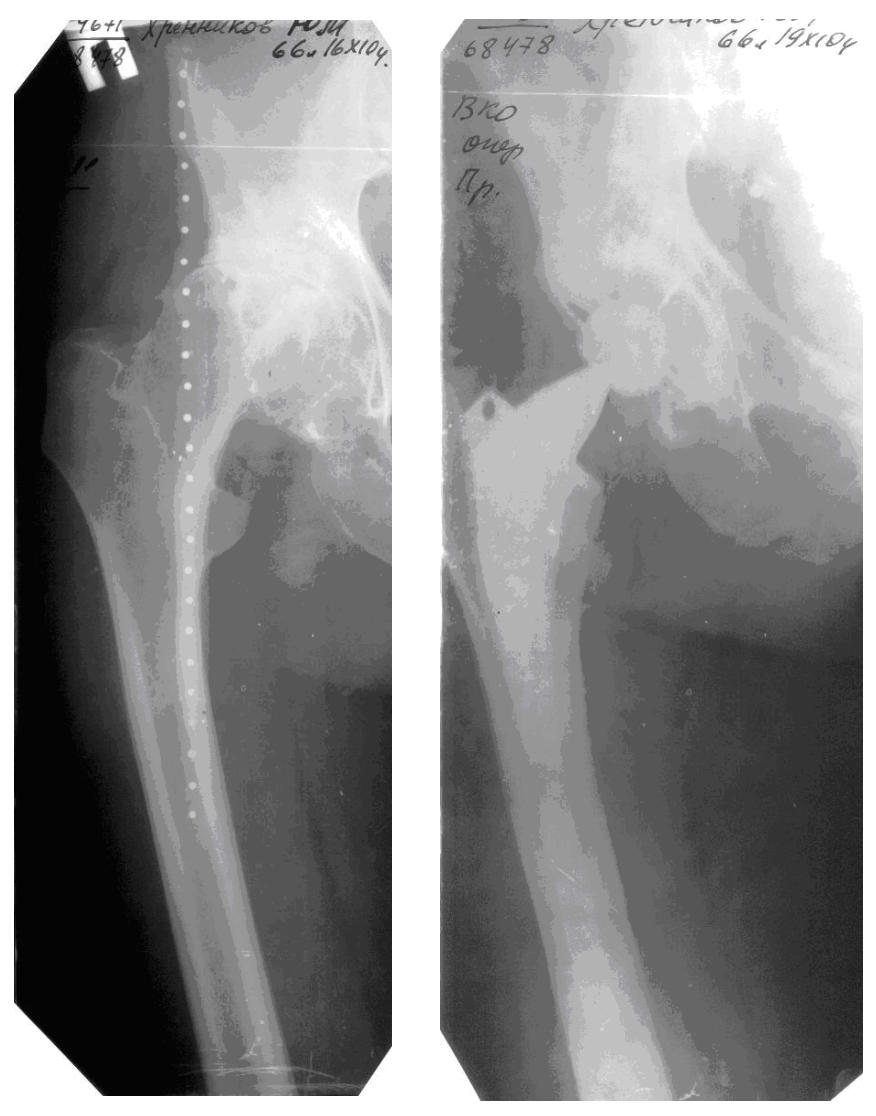

Figure 8. Röntgenogram of the joint before (left) and after (right) the operation.

\section{CONCLUSIONS}

A new construction of the hip joint using sapphire and metallic materials has been developed, and the methodology of the corresponding operations and surgical instrumentation was improved. Clinical and laboratory investigations of patients with hip joint pathology was performed to obtain more precise data. Clinical approval of the new endoprosthesis design, further evaluation of the results of the hip joint endoprosthetics and the filing of 
a patent for the novel elaboration of the artificial hip joint are under way.

The system described here is considerably more efficient than an ordinary implant, although the cost of the instruments, procedure and materials is similar. Moreover, many clinics have to repeat $30-50 \%$ of all of their hipimplantation operations because ordinary endoprostheses loosen and wear.

A strictly individual approach for the development and manufacturing of the bioengineering construction of endoprostheses has been developed, with rapid manufacturing of the individual components.

Smaller overall dimensions of stem and removal of a smaller part of the bone head for the implant allow the traumatic effects of preparation of the thigh bone channel to be reduced.

The novel construction of the stem makes provision for bionic implantation of bone tissue in the body of the stem, which prevents its loosening.

The use of a sapphire monocrystal for the production of the endoprosthetic spherical head reduces wear and prolongs longevity of the endoprosthetic system.

\section{ACKNOWLEDGMENTS}

A key partner in this work is the Scientific and Technological Concern "Institute for Monocrystals", Kharkov, Ukraine a leading research organization of the former USSR in the field of the development and application of methods of production of crystals and their products. The scientists and engineers who now deal with the development of sapphire endoprostheses already have experience in the creation of complicated products from high-temperature crystals and are able to produce the sapphire components. The growth, processing, baking and other crystal parameters have been investigated by the Corundum Monocrystal Technology Department, which has equipment for growing sapphire moncrystals (models "Crystal-ZM" and "Crystal-606"); quality control of monocrystals; and installations for crystal treatment in different ambient atmospheres (model SSHVL-2000).

\section{REFERENCES}

1. Kulish, N.I. \& Tankut, V.A. Surgical Operation in nonspecific arthritis of hip joints. In: XVI Symposium of the European Society of Osteoarthrolosis "Destruction of Joints", pp. 80-81. Moscow (1987).

2. Korzh, A.A., Kulish, N.I., Tankut, V.A. \& Mihailiv, V.T. Evolution of method of arthroplastics at koksartroze. Journal of Orthopedy, Traumatology and Prosthesis 10 (1990) 1-7.

3. Filipenko, V.A., Miteleva, Z.M., Petrenko, D.E. \& Konareva, N.M. Theoretical basis for the selection of cementless endoprosthesis stem of hip joint. Journal of Orthopedy, Traumatology and Prosthesis 2 (2003) 32-36.

4. Rybachuk, O.I. Errors and complications for total endoprosthesing of hip joint. Journal of Orthopedy, Traumatology and Prosthesis 2 (1997) 13-19.

5. Loskutov, A.E. \& Olejnik, A.E. Endoprosthesing of hip joint in case of aseptic necrosis of head of thigh-bone. Journal of Orthopedy, Traumatology and Prosthesis 2 (1998) 48-52.

6. Berry, D.J. \& Muller, M.E. Revision arthroplasty using antiprotrusio cage for massive acetabular bone deficiency. J. Bone Joint Surg. (Am) 74 (1992) 711-715.

7. Hakkanen, M., Corbet, S.A., Betten, J. et al. Aseptic loosening of total hip replacement. J. Bone Joint Surgery (Br) 79 (1997) 467-474.

8. Loskutov, A.E. \& Nikiforov, I.A. Endoprosthesis of hip joint. Condition of problem and tendency of development. Journal of Orthopedy, Traumatology and Prosthesis 1 (2002) 94-101.

9. Filipenko,V.A., Miteleva, Z.M., Petrenko, D.E. et al. Research of stressedly-deformed condition of system "endoprosthesisbone" with different degrees of their contact. Journal of Orthopedy, Traumatology and Prosthesis 3 (2003) 96-100.

10. Coombs, R., Gristina, A. \& Hungenborot, D. Joint Replacement, p. 47. New York: Wiley-Liss (1990).

11. Feinstein D.I., Alternative input/output data to CAD/CAM systems. In: 9th International and Computer Vision Users Conference, pp. 77-87. Detroit (1998).

12. Satava, R.M. Cybersurgery. Advanced Technologies for Surgical Practice, p. 201. New York: Wiley-Liss (1998).

13. Handels, H., Ehrhardt, J., Plotz, W. \& Poppl, S.J. Computerassisted planning and simulation of hip operations using virtual three-dimensional models. Stud. Health Technol. Inform. 68(1999) 686-689.

14. Ukraine patent for the invention "Hip Joint Endoprosthesis" UA №65197 and UA №6519. 